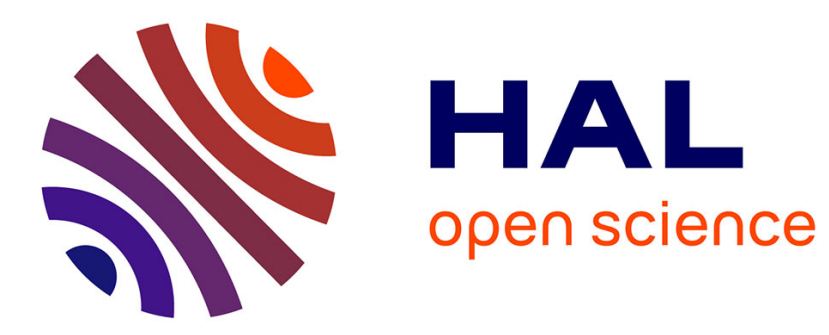

\title{
DCD and comorbidity in neurodevelopmental disorder: how to deal with complexity?
}

Bouwien C.M. Smits-Engelsman, Marianne’ Jover, Dido Green, Gillian Ferguson, Peter Wilson

\section{- To cite this version:}

Bouwien C.M. Smits-Engelsman, Marianne' Jover, Dido Green, Gillian Ferguson, Peter Wilson. DCD and comorbidity in neurodevelopmental disorder: how to deal with complexity?. Human Movement Science, 2017, 53, pp.1 - 4. 10.1016/j.humov.2017.02.009 . hal-01792681

\section{HAL Id: hal-01792681 \\ https://hal.science/hal-01792681}

Submitted on 15 May 2018

HAL is a multi-disciplinary open access archive for the deposit and dissemination of scientific research documents, whether they are published or not. The documents may come from teaching and research institutions in France or abroad, or from public or private research centers.
L'archive ouverte pluridisciplinaire HAL, est destinée au dépôt et à la diffusion de documents scientifiques de niveau recherche, publiés ou non, émanant des établissements d'enseignement et de recherche français ou étrangers, des laboratoires publics ou privés. 


\title{
DCD and comorbidity in neurodevelopmental disorder: how to deal with complexity?
}

\author{
Bouwien CM Smits-Engelsman ${ }^{\mathrm{a}}$, Marianne Jover ${ }^{\mathrm{b}}$, Dido Green ${ }^{\mathrm{c}}$, Gillian Ferguson ${ }^{\mathrm{a}}$ and Peter Wilson ${ }^{\mathrm{d}}$ \\ ${ }^{a}$ Department of Health \& Rehabilitation Sciences, Faculty of Health Sciences, University of Cape \\ Town, Cape Town, South Africa. \\ ${ }^{\mathrm{b}}$ PsyCLE, Aix Marseille Université, Aix en Provence, France. \\ ${ }^{\mathrm{c}}$ Centre for Rehabilitation, Oxford Brookes University, Oxford, UK. \\ ${ }^{\mathrm{d}}$ School of Psychology, Australian Catholic University, Australia.
}

In 1994, leading researchers in the field of Developmental Coordination Disorder (DCD) research gathered at the London International Consensus Conference in Canada for discussions on the nomenclature, description and definition, assessment and management of motor coordination problems in children. Since then, biannual international conferences on children with DCD have been held, becoming leading events to exchange and update information for researchers, clinicians and students, and also for parents and patients. The last International conference in DCD was held in Toulouse, France from the July 2 nd to 4 th, 2015. Each conference has covered a specific theme with the organizing committee proposing the following "Developmental coordination disorder and other neurodevelopmental disorders: a focus on comorbidity". Comorbidity refers to the presence of two or more disorders in the same individual. Co-morbidity in neurodevelopmental disorders is pronounced, (particularly DCD, dyslexia, autism spectrum disorders (ASD) and attention deficit hyperactivity disorder ADHD in the area of developmental disorders). Many studies suggest that as many as $50 \%$ of individuals diagnosed with a psychiatric or neurodevelopmental problem suffer in fact from more than one disorder (e.g. (Williams, 2013)\&Green \& Baird, 2005). Comorbidity or a cooccurring disorder seriously impacts outcomes and creates a significant constraint on family and school life. Furthermore, it complicates diagnostic procedures and organization of health care. The 11th congress on DCD aimed to provide some answers to the important questions of diagnostic criteria, causal factors, prognostic markers, management of DCD and associated disorders.

This conference was a real success and welcomed nearly 400 participants from 22 countries. The plenary sessions included keynote presentations focusing specifically on DCD (A. Kirby, D. Dewey, ML Kaiser and B. Smits-Engelsman) or more broadly on motor learning and comorbidity (M Habib, H Forssberg, R Nicolson), setting DCD into the broader area of neurodevelopmental disorders. Seventyeight oral presentations were distributed between 24 sessions addressing both fundamentals and applied topics: physical manifestations, perinatology, locomotion, perceptual motor integration, manual functions and handwriting, cognition, internal model, neural correlates, assessment, participation, physical activity, intervention adulthood, mental health. Poster sessions were an 
opportunity for students and young researchers to present, over the duration of the conference, on projects and ongoing research. Finally, workshops were held for professionals and parents wishing to have at their disposal different techniques available for DCD children.

Twenty-six papers were submitted for publication of which eight are included in this special section of HMS. Beside the three papers on instruments to assist diagnosis, remaining papers no longer focus on just describing the motor outcome but more on the interaction between children's characteristics (Executive Functioning), environmental aspects (stress) and every day tasks (play or crossing a road). It is important for clinicians as well as movement scientists to understand the full range of key constraints on each individual child with movement problems such as DCD during the acquisition of daily activities.

The dynamical systems theory implies that the acquisition of expertise emerges under the interaction of individual (structural and functional), task, and environmental constraints (Newell, 1986). It is clear from dynamical systems theory that the constraints on the acquisition of skills for each individual are many and interacting. Focusing on the interaction of constraints, offset by strengths, has several implications for the acquisition of skills in individual children. Four papers confirm this trend by reporting the interaction of motor development and the environment. The first paper, in identifying play characteristics of pre-school children with Developmental Coordination Disorder via parental questionnaires, elaborates on the relationship/interaction between play characteristics of children with DCD as well as the difficulties they experience in their ADL. Play is one of the most significant activities that affect children's development and insight into play characteristics of children with DCD may contribute to facilitating their daily participation.

The second paper (The relationship between motor proficiency and mental health outcomes in young adults: A test of the Environmental Stress Hypothesis (ESH)) aims to better understand the complex inter-relationship between motor proficiency, self-related concepts (like self-esteem and self-efficacy) and psychological adjustment, which have gathered momentum over the past 15 years. The ESH is arguably the most widely cited account of these relationships in the recent literature. In this study, Rigoli and colleagues test this hypothesis in young adults. Results showed that perceived social support was a significant mediating factor in the relationship between motor proficiency and internalizing symptoms, while physical self worth was not. While a relatively small scale modeling study, these results affirm the importance of social support in the mental health of young adults with movement problems. The data are instructive for clinicians as they seek to leverage their interventions, impacting other areas of development and well-being.

In light of recent evidence on the presence of executive function (EF) deficits in school-age children with DCD (Wilson, 2013) the third study of Houwen and colleagues (The Relationship Between 
Motor Performance and Parent-Rated Executive Functioning in 3- to 5-year-old Children: What is the Role of Confounding Variables) is a timely extension, addressing the relationship between motor performance and EF in preschoolers. EF was assessed here using a parent report scale (the Behavior Rating Inventory of Executive Function-Preschool version; BRIEF-P) while movement skill was measured using the MABC-2. Significant correlations between BRIEF sub-scales (i.e., Working memory, Planning/Organisation and Inhibition) and MABC scores were weak to moderate, at best. Interestingly, after controlling for age, gender, SES, and ADHD symptoms, only for working memory was the predictive relationship maintained. Taken together, the results show a complex pattern of relationship between motor and cognitive functions. Importantly, results do highlight that selective deficits in EF are already present in preschool children with motor coordination difficulties, suggesting the need for comprehensive assessment and careful adjustments to the treatment of DCD in these young children.

The fourth paper (The Use of Visually Guided Behaviour in Children with Developmental Coordination Disorder (DCD) when Crossing a Virtual Road) explores the interaction between children with DCD and their physical environment. Specifically, the interaction between visually guided behavior and children's vulnerability at the roadside is discussed. The authors assessed the ability of both typically developing and children with DCD to select suitable temporal crossing gaps in the context of road crossing in a virtual environment.

In this issue, three papers address the important issue of the validity of assessments across cultures (Vincon; Peritta) and contexts lifespan (Parker). Vincon et al have used data acquired from the German standardization of the Bruininks-Oseretsky Test of Motor Proficiency - 2nd Edition (BOT-2), to consider the extent to which a test performance reflects the skills of children in everyday life. Hypotheses regarding the underlying components of a task were not necessarily upheld with balance and bilateral coordination items of the BOT-2 not showing an association with bike riding or sports. Thus, the gestalt of skills in daily life is not just greater than the sum, but perhaps qualitatively as well as quantitatively different.

The second paper (Parker et al.: Standardised motor assessment in young adults: the Bruininks Oseretsky Test-2 Short Form and the McCarron Assessment of Neuromuscular Development) advances our understanding of motor impairment in young adults. One of the reasons this area remains under-researched is due to the limited availability of valid assessment instruments. This paper explores the issue regarding two commonly used tests in this age-group. With DSM-5 extending diagnostic criteria to cater for this older group some guidance is warranted on the 
standardised assessment options. The authors recommend new approaches using ecologically valid tasks.

The last paper (Peritta el al.,) reports on the reliability and validity of the Finnish version of the Motor Observation Questionnaire for Teachers. Methods that enable teachers to identify children with DCD in a clear and unambiguous manner are vitally important. As an initial screening tool, observational checklists are a very useful addition to the assessment regime. Motivated by this issue, the authors examined the psychometric qualities of a Finnish adaptation of the MOQ-T-FI, designed originally for Dutch children. For a large sample of children ( $n=193)$ aged six to 12 years, results were broadly consistent with those for Dutch and Italian children. Using confirmatory factor analysis, a bifactorial model provided the best solution: a single higher level (motor) factor was revealed, with two lower (or specific) factors labelled motor functioning and handwriting. However, not all handwriting items loaded cleanly onto the relevant/specific factor. Specificity and sensitivity metrics for the MOQ-T-FI were mixed, and concurrent validity (against the MABC) was moderate, only. This underscores the need for additional validation data. Notwithstanding this, the scale has promise and, with refinement may serve several functions, as asserted by the authors: (1) raising teacher awareness of movement skill issues in children; (2) aiding the identification of children at risk for DCD, and (3) providing a profile of functional limitations across tasks.

\section{New trends and future research}

There is accumulating evidence for biological and brain based origins for DCD by using a wide array of neuroimaging techniques, electroencephalography (EEG), and transcranial ultrasound (for reviews, see (Brown-Lum, 2015) (Peters, 2013); (Zwicker, 2012)). It has also become clear that there is partly a genetic basis for DCD ( (Mosca, 2016)) which, in interaction with reduced opportunities to practice new movement skills, may lead to significant longer term issues in functional performance. Genetic loci implicated in DCD (e.g., GAP43, RBFOX1 and other genes) are linked to a range of neurodevelopmental disorders and are strongly implicated in the maturation and function of neural networks previously associated with DCD. Genetic risk factors, combined with reduced exposure to motor learning experiences - either by virtue of socioeconomic disadvantage or other reason - may severely impact the child's capacity to acquire new skills or their level of motor performance (Lingam, 2009). A countervailing factor identified by clinicians is the child's psychological predispositions including their goal motivation and ability to persevere when learning new skills, even when learning trajectories are perhaps shallower than is typical. Whatever the child's make-up, the ability of instructors to provide a safe and enjoyable context for learning is critical to the long 
term success of training. Where co-occurring issues are also evident (e.g. poor executive function, planning or attentional difficulties), this is even more important. Practice might not necessarily make perfect in this group of children, but positive learning experiences and skilled instruction will give the child with DCD a better chance of success.

Heterogeneity in the expression of DCD, the presence of co-occurring problems, and perhaps its aetiology suggests that we move beyond a 'one-size-fits-all' approach to assessment and intervention. What remains to be shown is whether assessments should be adjusted to account for different co-morbidities. For example, if a task instruction is to perform an activity "as quickly and accurately as possible, will a child with ADHD focus on the first, second or both parts of the instruction? The outcome would be influenced by the child's processing and memory constraints, among other factors. The different effects of implicit and explicit instructions in assessment has been shown in children with Autism who perform worse on 'timed' tasks compared with criterion based ones on the Movement Assessment Battery for Children (Green D. C., 2009). Revising the instruction, for example, to "stand on one leg until the sand has moved from the top to the bottom" (using a 10 or $20 \mathrm{~s}$ timer) may provide a more tangible indicator of 'time' than the instruction to stand 'as long as you can'. In terms of the process of motor learning, such instructions may also help the learner attend to the (external) effects of their actions, rather than to vague or internally referenced aspects of the movement. Exploring issues related varying ways of instructions for clinical assessments and intervention has yet to be explored but provides an interesting avenue for research. Moreover studies that ascertain which characteristics intervention enhances sensorimotor learning best are urgently needed because children with $D C D$ may responds differently to training variables.

The complexity of heterogeneity and frequency of co-morbid/co-occurring conditions within DCD presents further challenges for intervention. This is reflected in the limited number of papers concerning interventions for children with complex comorbidities. It is unlikely that a single intervention could ever be appropriate for this group, especially when considering the transactional elements between child, task and environment. Mapping profiles of performance across multiple cognitive and behavioral profiles, defining strengths as well as 'impairments' should be the way forward in determining appropriate interventions. While intervention studies remain limited, contrasting profiles of the participating individuals against recognized subtypes (such as (Green D. C., 2008; Macnab, 2001; Hoare, 1994; Vaivre-Douret, 2011) may provide a useful framework to progress our understanding of intervention efficacy; especially in the absence of funding for large scale intervention studies that can account for the multiple and variable factors of variation within DCD, common association with other neurodevelopmental disorders and environmental and personal factors. Lastly because applying knowledge gained in learning in normal development may not be 
straightforwardly translated to children with DCD, studies on motor skills learning (internal versus external focus; implicit versus explicit learning; variable versus constant training) should be repeated in these specific DCD groups (young vs older; with and without co-morbidity). For instance doing many different tasks in one session may be good of typically developing but overwhelming for children who have motor problems or distracting in children with DCD/ADHD.

Lastly defining interventions and the salient ingredients of these in enough detail are essential to our understanding of treatment response and the possibility to apply knowledge towards new research and implement within clinical practice.

As yet, and importantly, as indicated by the number of papers presented the conference, is the impact of and on mental health in DCD. The DCDXI conference highlighted these issues together with the increase in number of papers on this issue from recent research. We welcome the increased focus on this very important area of development.

\section{References}

Brown-Lum, M. Z. (2015). Brain Imaging Increases our Understanding of Developmental Coordination Disorder: a Review of Literature and Future Directions. Current Developmental Disorders Reports, 131-140.

Green, D. C. (2008). Does Subtype of Developmental Coordination Disorder Count: Is there a Differential Effect on Outcome Following Intervention? . Human Movement Science, 363-382.

Green, D. C. (2009). Impairment in Movement Skills of Children with Autistic Spectrum Disorders. Developmental Medicine \& Child Neurology, 311-316.

Hoare, D. (1994). Subtypes of Developmental Coordination Disorder. Adapted Physical Activity Quarterly, 158-169.

Lingam, R. H. (2009). Prevalence of Developmental Coordination Disorder using the DSM-IV at 7 years of Age: a UK Population-Based Study. Pediatrics, 693-700.

Macnab, J. M. (2001). The search for Subtypes of DCD: Is Cluster Analysis the Answer? Human Movement Science, 49-72.

Mosca, S. J. (2016). Copy-Number Variations are enriched for Neurodevelopmental Genes in Children with Developmental Coordination Disorder. Journal of Medical Genetics, 812-819.

Newell, K.M. (1986). Constraints on the development of coordination. In: M.G. Wade, \& H.T.A. Whiting (Eds). Motor development in children: aspects of coordination and control (pp. 34161) Amsterdam: Martin Nijhoff. 
Peters, L. M.-A. (2013). Neural Correlates of Developmental Coordination Disorder. Developmental Medicine \& Child Neurology, 59-64.

Vaivre-Douret, L. L.-M. (2011). Subtypes of Developmental Coordination Disorder: Research on their Nature and Etiology. Dev Neuropsychol, 614-643.

Williams, D. M. (2013). Comorbidity and Diagnosis of Developmental Disorders. Current Issues in Developmental Disorders, 19-45.

Wilson, P. R.-E. (2013). Understanding Performance Deficits in Developmental Coordination Disorder: a Meta-Analysis of recent Research. Dev Med Child Neurol., 217-228.

Zwicker, J. M. (2012). Developmental Coordination Disorder: a Pilot Diffusion Tensor Imaging Study. Pediatric Neurology, 162-167. 